\title{
SAINT-VENANT'S PROBLEM FOR COMPOSITE MICROPOLAR ELASTIC CYLINDERS
}

\author{
D. IEŞAN and S. CHIRITA \\ University of Iaşi, Iaşi-Romania
}

\begin{abstract}
We consider Saint-Venant's problem in the case when the cross-section of the cylinder is occupied by different inhomogeneous and isotropic micropolar elastic solids. The results are used to study the deformation of a circular composite cylinder.
\end{abstract}

\section{INTRODUCTION}

THE DEFORMATION of homogeneous and isotropic micropolar elastic cylinders was studied in various papers $[1-6]$. In this paper we consider the case of a composite cylinder when the generic cross-section is occupied by different inhomogeneous and isotropic micropolar elastic solids. We assume that the elastic coefficients are independent of the axial coordinate. In the classical theory of elasticity this problem was considered in $[7,8]$.

In the first part of the paper we present a method of solving Saint-Venant's problem. Then, we use this method to study the deformation of a composite circular cylinder.

\section{STATEMENT OF THE PROBLEM}

Throughout this paper $R$ denotes the interior of a right cylinder of the length $l$ with the generic cross-section $\Sigma$ and the lateral boundary $B$. We call $\partial R$ the boundary of $R$, and designate by $n_{i}$ the components of the outward unit normal of $\partial R$. Throughout this paper a rectangular Cartesian coordinate system $O x_{k}(k=1,2,3)$ is used. The rectangular Cartesian coordinate frame is chosen such that the $x_{3}$-axis is parallel to the generators of $R$ and the $x_{1} O x_{2}$-plane contains one of the terminal cross-sections. We call $\Sigma^{(o)}$ the cross-section located at $x_{3}=o$ and $\Sigma^{(i)}$ the cross-section which lies in the plane $x_{3}=l$. We denote by $L$ the boundary of the generic cross-section $\Sigma$.

We shall employ the usual summation and differentiation conventions: Greek subscripts are understood to range over the integers $(1,2)$ whereas Latin subscripts-over $(1,2,3)$; summation over repeated subscripts is implied and subscripts preceded by a comma denote differentiation with respect to the corresponding Cartesian coordinate.

In this paper we consider the linear theory of micropolar elasticity. The basic equation for isotropic and centrosymmetric solids are [9]:

Equilibrium equations, in absence of body forces and body couples

$$
t_{i j, j}=0, \quad m_{j i, j}+\epsilon_{i r s} t_{r s}=0,
$$

constitutive equations

$$
t_{i j}=\lambda e_{r} \delta_{i j}+(\mu+\kappa) e_{i j}+\mu e_{j i}, \quad m_{i j}=\alpha \varphi_{r, r} \delta_{i j}+\beta \varphi_{i, j}+\gamma \varphi_{j, i},
$$

geometrical equations

$$
e_{i j}=u_{j, i}+\epsilon_{j i r} \varphi_{r}
$$

In these equations we have used the following notations: $t_{i j}$, components of the stress tensor; $m_{i j}$, components of the couple-stress tensor; $u_{i}$, components of the displacement vector; $\varphi_{i}$, components of the microrotation vector; $e_{i j}$, components of the strain tensor; $\delta_{i j}$, Kronecker's delta; $\epsilon_{i j k}$, alternating symbol; $\lambda, \mu, \kappa, \alpha, \beta, \gamma$, characteristic coefficients of the material. 
We assume that $\Sigma$ is a $C^{1}$-smooth domain [10]. Let $L_{1}$ and $L_{2}$ be two disjoint subsets of $L$ such that $L=L_{1} \cup L_{2}$. Let $\Gamma$ be a curve contained in $\Sigma$ satisfying the conditions that $L_{\rho} \cup \Gamma$ $(\rho=1,2)$ is the boundary of a domain $\Sigma_{\rho}$ contained in $\Sigma$ such that $\Sigma_{1} \cap \Sigma_{2}=\emptyset$.

Suppose that $\Sigma_{1}$ and $\Sigma_{2}$ are occupied by two elastic materials. Let $\lambda^{(\rho)}, \mu^{(\rho)}, \kappa^{(\rho)}, \alpha^{(\rho)}, \beta^{(\rho)}$, $\gamma^{(\rho)}$ be the elastic coefficients relative to $\Sigma_{\rho}$. We denote by $R_{\rho}$ the domain occupied by the material with the elastic coefficients $\lambda^{(\rho)}, \ldots, \gamma^{(\rho)}$. Throughout this paper we assume that

$$
\lambda^{(\rho)}=\lambda^{(\rho)}\left(x_{1}, x_{2}\right), \mu^{(\rho)}=\mu^{(\rho)}\left(x_{1}, x_{2}\right), \ldots, \gamma^{(\rho)}=\gamma^{(\rho)}\left(x_{1}, x_{2}\right) .
$$

The functions $\lambda^{(p)}, \ldots, \gamma^{(p)}$ are supposed to belong to $C^{\infty}$ and the elastic potential corresponding to the body which occupies $R_{\rho}$ is assumed to be a positive definite quadratic form.

We can consider $\Sigma$ as being occupied by an elastic medium which, in general, has elastic coefficients discontinuous along $\Gamma$. We denote by $\Pi$ the surface of separaticn of the two materials.

The functions $u_{i}, \varphi_{i}$ must be continuous in passing from one medium to another so that we have the conditions

$$
\begin{gathered}
{\left[u_{i}\right]_{1}=\left[u_{i}\right]_{2},\left[\varphi_{i}\right]_{1}=\left[\varphi_{i}\right]_{2},} \\
{\left[t_{\beta i}\right]_{1} \nu_{\beta}=\left[t_{\beta i}\right]_{2} \nu_{\beta},\left[m_{\beta i}\right]_{1} \nu_{\beta}=\left[m_{\beta i}\right]_{2} \nu_{\beta}, \quad \text { on } \Pi,}
\end{gathered}
$$

where it has been indicated that the expressions in the parentheses are calculated for the domains $R_{1}$ and $R_{2}$, respectively, and $\nu_{\beta}$ are the direction cosines of the vector normal to $\Gamma$, outward to $\Sigma_{1}$.

The cylinder is supposed to be free of lateral loading so that we have the conditions

$$
t_{\alpha i} n_{\alpha}=0, m_{\alpha i} n_{\alpha}=0 \text {, on } B \text {. }
$$

Saint-Venant's problem consists in determining the equilibrium of the cylinder (in absence of body forces and body couples) when the load applied to one of the bases of the cylinder is statically equivalent to a force $P\left(P_{i}\right)$ and a couple $M\left(M_{i}\right)$ and is free of lateral loading.

We will have occasion to use three special problems $P^{(s)}(s=1,2,3)$ of plane strain. In what follows we denote by $u_{\alpha}^{(s)}, \varphi_{3}^{(s)}, e_{i j}^{(s)}, t_{i j}^{(s)}, m_{i j}^{(s)}$ the functions $u_{\alpha}, \varphi_{3}, e_{i j}, t_{i j}, m_{i j}$ for the problems $P^{(s)}$.

The problems $P^{(s)}$ are characterized by the equations

$$
\begin{gathered}
t_{\beta \alpha, \beta}^{(\eta)}+\left(\lambda^{(\rho)} x_{\eta}\right)_{, \alpha}=0, t_{\beta \alpha, \beta}^{(3)}+\lambda_{. \alpha}^{(\rho)}=0, \\
m_{\beta, \beta}^{(\eta)}+\epsilon_{3 \alpha \beta} t_{\alpha \beta}^{(\eta)}+\epsilon_{3 \alpha \eta} \beta_{, \alpha}^{(\rho)}=0, m_{\beta}^{(3), \beta}+\epsilon_{3 \alpha \beta} t_{\alpha \beta}^{(3)}=0, \\
t_{\alpha \beta}^{(s)}=\lambda^{(\rho)} e_{\eta \eta}^{(s)} \delta_{\alpha \beta}+\left(\mu^{(\rho)}+\kappa^{(\rho)}\right) e_{\alpha \beta}^{(s)}+\mu^{(\rho)} e_{\beta \alpha}^{(s)}, m_{\alpha}^{(s)}=\gamma^{(\rho)} \varphi_{3, \alpha}^{(s)}, \\
\left.e_{\alpha \beta}^{(s)}=u_{\beta, \alpha}^{(s)}+\epsilon_{3 \beta \alpha} \varphi\right\}^{(s)}, \text { in } \Sigma_{\rho,}
\end{gathered}
$$

and the following conditions

$$
\begin{gathered}
\left.\left[u_{\alpha}^{(s)}\right]_{1}=\left[u_{\alpha}^{(s)}\right]_{2},[\varphi\}^{(s)}\right]_{1}=\left[\varphi_{3}^{(s)}\right]_{2},\left[t_{\beta \alpha}^{(s)}\right]_{1} \nu_{\beta}=\left[t_{\beta \alpha}^{(s)}\right]_{2} \nu_{\beta}+p_{\alpha}^{(s)}, \\
{\left[m_{\alpha}^{(s)}\right]_{1} \nu_{\alpha}=\left[m_{\alpha}^{(s)}\right]_{2} \nu_{\alpha}+q^{(s)}, \text { on } \Gamma,} \\
{\left[t_{\beta \alpha}^{(n)} n_{\beta}\right]_{\rho}=-\lambda^{(\rho)} x_{\eta} n_{\alpha},\left[t_{\beta \alpha}^{(3)} n_{\beta}\right]_{\rho}=-\lambda^{(\rho)} n_{\alpha},\left[m_{\alpha}^{(\eta)} n_{\alpha}\right]_{\rho}=\epsilon_{3 \eta \nu} \beta^{(\rho)} n_{\nu},} \\
{\left[m_{\alpha 3}^{(3)} n_{\alpha}\right]_{\rho}=0, \text { on } L_{\rho}}
\end{gathered}
$$

where

$$
\begin{gathered}
p_{\alpha}^{(\eta)}=\left(\lambda^{(2)}-\lambda^{(1)}\right) x_{\eta} \nu_{\alpha}, p_{\alpha}^{(3)}=\left(\lambda^{(2)}-\lambda^{(1)}\right) \nu_{\alpha}, \\
q^{(\eta)}=\epsilon_{3 \eta \alpha}\left(\beta^{(1)}-\beta^{(2)}\right) \nu_{\alpha}, q^{(3)}=0 .
\end{gathered}
$$


It is easy to prove that the necessary and sufficient conditions for the existence of the solution are satisfied for each boundary value problem $P^{(s)}$. In what follows we assume that the functions $u_{a}^{(s)}, \varphi_{3}^{(s)}$ are known.

\section{EXTENSION AND BENDING BY TERMINAL COUPLES}

Let the loading applied on the end $\Sigma^{(o)}$ be statically equivalent to the force $P\left(o, o, P_{3}\right)$ and the moment $M\left(M_{1}, M_{2}, o\right)$. Thus, for $x_{3}=o$ we have the following conditions

$$
\begin{gathered}
\int_{\Sigma} t_{3 \alpha} \mathrm{d} \sigma=0, \\
\int_{\Sigma} t_{33} \mathrm{~d} \sigma=-P_{3}, \\
\int_{\Sigma}\left(x_{\alpha} t_{33}-\epsilon_{3 \alpha \beta} m_{3 \beta}\right) \mathrm{d} \sigma=\epsilon_{3 \alpha \beta} M_{\beta}, \\
\int_{\Sigma}\left(\epsilon_{3 \alpha \beta} x_{\alpha} t_{3 \beta}+m_{33}\right) \mathrm{d} \sigma=0 .
\end{gathered}
$$

The problem consists in the solving of the eqns (2.1)-(2.3) with the conditions (2.5), (2.6), (3.1)-(3.4). We seek the solution in the form

$$
\begin{aligned}
& u_{\alpha}=-\frac{1}{2} a_{\alpha} x_{3}^{2}+\sum_{s=1}^{3} a_{s} u_{\alpha}^{(s)}, u_{3}=\left(a_{1} x_{1}+a_{2} x_{2}+a_{3}\right) x_{3}, \varphi_{\alpha}=\epsilon_{3 \alpha \beta} a_{\beta} x_{3}, \\
& \varphi_{3}=\sum_{s=1}^{3} a_{s} \varphi_{3}^{(s)}
\end{aligned}
$$

where $u_{\alpha}^{(s)}, \varphi_{3}^{(s)}$ are the solutions of the problems $P^{(s)}$ and $a_{s}$ are unknown constants. From (3.5) and (2.2) we get

$$
\begin{aligned}
& t_{\alpha \beta}=\lambda^{(\rho)}\left(a_{1} x_{1}+a_{2} x_{2}+a_{3}\right) \delta_{\alpha \beta}+\sum_{s=1}^{3} t_{\alpha \beta}^{(s)} \\
& t_{33}=\left(\lambda^{(\rho)}+2 \mu^{(\rho)}+\kappa^{(\rho)}\right)\left(a_{1} x_{1}+a_{2} x_{2}+a_{3}\right)+\lambda^{(\rho)} \sum_{s=1}^{3} a_{s} e_{\alpha \alpha}^{(s)} \\
& t_{\alpha 3}=t_{3 \alpha}=0, m_{\alpha \beta}=m_{33}=0 \\
& m_{\alpha 3}=\epsilon_{3 \alpha \nu} \beta^{(\rho)} a_{\nu}+\sum_{s=1}^{3} a_{s} m_{\alpha}^{(s)} \\
& m_{3 \alpha}=\epsilon_{3 \alpha \nu} \gamma^{(\rho)} a_{\nu}+\sum_{s=1}^{3} a_{s} m_{3 \alpha}^{(s)} .
\end{aligned}
$$

Using (2.7)-(2.11), (3.5) and (3.6) it follows that the conditions (2.5) and (2.6) are satisfied.

The conditions (3.1) and (3.4) are satisfied on the basis of the relations (3.6). From (3.2), (3.3) and (3.6) we obtain the following system for the unknown constants

$$
A_{r s} a_{s}=B_{r}
$$

where

$$
\begin{aligned}
& A_{\alpha \beta}=\sum_{\rho=1}^{2} \int_{\Sigma_{\rho}}\left\{x_{\alpha}\left[\left(\lambda^{(\rho)}+2 \mu^{(\rho)}+\kappa^{(\rho)}\right) x_{\beta}+\lambda^{(\rho)} e_{\eta \eta}^{(\beta)}\right]-\epsilon_{3 \alpha \lambda}\left(\epsilon_{3 \lambda \beta} \gamma^{(\rho)}+m_{3 \lambda}^{(\beta)}\right)\right\} \mathrm{d} \sigma \\
& A_{\alpha 3}=\sum_{\rho=1}^{2} \int_{\Sigma_{\rho}}\left\{x_{\alpha}\left[\lambda^{(\rho)}+2 \mu^{(\rho)}+\kappa^{(\rho)}+\lambda^{(\rho)} e_{\eta \eta}^{(3)}\right]-\epsilon_{3 \alpha \lambda} m_{3 \lambda}^{(3)}\right\} \mathrm{d} \sigma
\end{aligned}
$$




$$
\begin{aligned}
& A_{3 \alpha}=\sum_{\rho=1}^{2} \int_{\Sigma_{\rho}}\left[\left(\lambda^{(\rho)}+2 \mu^{(\rho)}+\kappa^{(\rho)}\right) x_{\alpha}+\lambda^{(\rho)} e_{\eta \eta}^{(\alpha)}\right] \mathrm{d} \sigma \\
& A_{33}=\sum_{\rho=1}^{2} \int_{\Sigma_{\rho}}\left[\lambda^{(\rho)}+2 \mu^{(\rho)}+\kappa^{(\rho)}+\lambda^{(\rho)} e_{\eta \eta}^{(3)}\right] \mathrm{d} \sigma \\
& B_{\alpha}=\epsilon_{3 \alpha \beta} M_{\beta}, B_{3}=-P_{3} .
\end{aligned}
$$

As in $[7,8]$ we can prove that $\operatorname{det}\left(A_{r s}\right) \neq o$ so that the system (3.7) determines the constants $a_{s}$.

\section{TORSION}

Let the loading applied on the end $\Sigma^{(o)}$ be statically equivalent to the force $P(o, o, o)$ and the moment $M\left(o, o, M_{3}\right)$. In this case for $x_{3}=o$, we have the following conditions

$$
\begin{gathered}
\int_{\Sigma} t_{3 \alpha} \mathrm{d} \sigma=0, \\
\int_{\Sigma} t_{33} \mathrm{~d} \sigma=0, \\
\int_{\Sigma}\left(x_{\alpha} t_{33}-\epsilon_{3 \alpha \beta} m_{3 \beta}\right) \mathrm{d} \sigma=0, \\
\int_{\Sigma}\left(\epsilon_{3 \alpha \beta} x_{\alpha} t_{3 \beta}+m_{33}\right) \mathrm{d} \sigma=-M_{3} .
\end{gathered}
$$

The problem consists in the solving of the eqns (2.1)-(2.3) with the conditions (2.5), (2.6) and (4.1)-(4.4). Using the results of [1] we try to solve the problem assuming that

$$
u_{\alpha}=\epsilon_{3 \beta \alpha} \tau x_{\beta} x_{3}, u_{3}=\tau \Phi\left(x_{1}, x_{2}\right), \varphi_{a}=\tau \Phi_{\alpha}\left(x_{1}, x_{2}\right), \varphi_{3}=\tau x_{3},
$$

where $\Phi, \Phi_{1}, \Phi_{2}$ are unknown functions and $\tau$ is a unknown constant.

Let $V=\left(G, G_{1}, G_{2}\right)$ be an ordered triplet of functions $G, G_{1}, G_{2}$. We introduce the notations

$$
\begin{aligned}
T_{\alpha} V= & \left(\mu^{(\rho)}+\kappa^{(\rho)}\right) G_{, \alpha}+\kappa^{(\rho)} \epsilon_{3 \alpha \beta} G_{\beta}, \\
S_{\alpha} V= & \mu^{(\rho)} G_{, \alpha}+\kappa^{(\rho)} \epsilon_{3 \beta \alpha} G_{\beta}, \\
M_{\alpha \beta} V= & \alpha^{(\rho)} G_{\nu, \nu} \delta_{\alpha \beta}+\beta^{(\rho)} G_{\alpha, \beta}+\gamma^{(\rho)} G_{\beta, \alpha} \\
\mathscr{L}^{(\rho)} V \equiv & \left(T_{\alpha} V\right)_{, \alpha}=\left[\left(\mu^{(\rho)}+\kappa^{(\rho)}\right) G_{, \alpha}\right]_{, \alpha}+\epsilon_{3 \alpha \beta}\left(\kappa^{(\rho)} G_{\beta}\right)_{. \alpha} \\
\mathscr{L}_{\nu}^{(\rho)} V \equiv & \left(M_{\beta \nu} V\right)_{, \beta}+\epsilon_{3 \nu \beta}\left(T_{\beta} V-S_{\beta} V=\left(\alpha^{(\rho)} G_{\lambda, \lambda}\right)_{, \nu}+\left(\beta^{(\rho)} G_{\lambda, \nu}\right)_{, \lambda}\right. \\
& +\left(\gamma^{(\rho)} G_{\nu, \lambda}\right)_{, \lambda}+\epsilon_{\nu \beta 3} \kappa^{(\rho)} G_{, \beta}-2 \kappa^{(\rho)} G_{\nu} \\
\mathscr{B}^{(\rho)} V \equiv & \left(T_{\alpha} V\right) n_{\alpha}=\left(\mu^{(\rho)}+\kappa^{(\rho)}\right) G_{, \alpha} n_{\alpha}+\kappa^{(\rho)} \epsilon_{3 \alpha \beta} G_{\beta} n_{\alpha}, \\
\mathscr{B}_{\nu}^{(\rho)} V \equiv & \left(M_{\alpha \nu} V n_{\alpha}=\alpha^{(\rho)} G_{\lambda, \lambda} n_{\nu}+\beta^{(\rho)} G_{\lambda, \nu} n_{\lambda}+\gamma^{(\rho)} G_{\nu, \lambda} n_{\lambda} .\right.
\end{aligned}
$$

Taking into account (2.2), (2.3) and (4.5) we obtain

$$
\begin{aligned}
t_{\alpha \beta} & =0, t_{33}=0, \\
t_{\alpha 3} & =\tau\left(T_{\alpha} \Lambda+\mu^{(\rho)} \epsilon_{3 \beta \alpha} x_{\beta}\right), \\
t_{3 \alpha} & =\tau\left[S_{\alpha} \Lambda+\left(\mu^{(\rho)}+\kappa^{(\rho)}\right) \epsilon_{3 \beta \alpha} x_{\beta}\right], \\
m_{\eta \nu} & =\tau\left(M_{\eta \nu} \Lambda+\alpha^{(\rho)} \delta_{\eta \nu}\right), m_{\alpha 3}=m_{3 \alpha}=0, \\
m_{33} & =\tau\left(\alpha^{(\rho)} \Phi_{\nu, \nu}+\alpha^{(\rho)}+\beta^{(\rho)}+\gamma^{(\rho)}\right),
\end{aligned}
$$

where $\Lambda=\left(\Phi, \Phi_{1}, \Phi_{2}\right)$. 
The equilibrium eqns (2.1) reduce to

$$
\mathscr{L}^{(\rho)} \Lambda=\epsilon_{\alpha \beta 3}\left(\mu^{(\rho)} x_{\beta}\right)_{, \alpha}, \mathscr{L}_{\nu}^{(\rho)} \Lambda=x_{\nu} \kappa^{(\rho)}-\alpha_{, \nu}^{(\rho)}, \text { in } \Sigma_{\rho},
$$

and the boundary conditions $(2.6)$ become

$$
\mathscr{B}^{(\rho)} \Lambda=\mu^{(\rho)} \epsilon_{3 \alpha \beta} x_{\beta} n_{\alpha}, \mathscr{B}_{\nu}^{(\rho)} \Lambda=-\alpha^{(\rho)} n_{\nu}, \quad \text { on } L_{\rho}
$$

The conditions (2.5) reduce to

$$
\begin{gathered}
{[\Phi]_{1}=[\Phi]_{2},\left[\Phi_{\alpha}\right]_{1}=\left[\Phi_{\alpha}\right]_{2},} \\
{\left[\mathscr{B}^{(1)} \Lambda\right](\nu)-\left[\mathscr{B}^{(2)} \Lambda\right](\nu)=\epsilon_{\alpha \beta 3}\left(\mu^{(1)}-\mu^{(2)}\right) x_{\beta} \nu_{\alpha},} \\
{\left[\mathscr{B}_{\eta}^{(1)} \Lambda\right](\nu)-\left[\mathscr{B}_{\eta}^{(2)} \Lambda\right](\nu)=\left(\alpha^{(2)}-\alpha^{(1)}\right) \nu_{\eta}, \text { on } \Gamma,}
\end{gathered}
$$

where $\left[\mathscr{B}^{(\rho)} \Lambda\right](\nu)$ denotes the operator $\mathscr{B}^{(\rho)} \Lambda$ for $n_{\alpha}=\nu_{\alpha}$.

Following $[10,11]$ we can prove that the necessary and sufficient conditions for the existence of the solution of the boundary value problem (4.10)-(4.12) is

$$
\sum_{\rho=1}^{2}\left[\int_{\Sigma_{\rho}} \mathscr{L}^{(\rho)} \Lambda \mathrm{d} \sigma-\int_{L_{\rho}} \mathscr{B}^{(\rho)} \Lambda \mathrm{d} s\right]-\int_{\Gamma}\left(\mathscr{B}^{(1)} \Lambda-\mathscr{B}^{(2)} \Lambda\right) \mathrm{d} s=0 .
$$

It is easy to see that this condition is satisfied. We assume that the functions $\Phi, \Phi_{\alpha}$ are known.

Taking into account (4.9) it follows that the conditions (4.2) and (4.3) are satisfied. As in [1] we can prove that the conditions (4.1) are identically satisfied. From (4.4) we determine the constant $\tau$. Using (4.9) the condition (4.4) reduce to

$$
\tau D=-M_{3},
$$

where $D$ is the torsional rigidity

$$
\begin{aligned}
D= & \sum_{\rho=1}^{2} \int_{\Sigma_{\rho}}\left(\epsilon_{3 \mu \rho} x_{\alpha}\left\{\mu^{(\rho)} \Phi_{, \beta}+\epsilon_{\nu \beta 3}\left[\kappa^{(\rho)} \Phi_{\nu}+\left(\mu^{(\rho)}+\kappa^{(\rho)}\right) x_{\nu}\right]\right\}\right. \\
& \left.+\alpha^{(\rho)} \Phi_{\nu, \nu}+\alpha^{(\rho)}+\beta^{(\rho)}+\gamma^{(\rho)}\right) \mathrm{d} \sigma .
\end{aligned}
$$

As in [1] we can prove that $D \neq o$. The relation (4.15) determines the constant $\tau$. Thus the torsion problem is solved.

\section{FLEXURE}

Let the loading applied on the end $\Sigma^{(o)}$ be statically equivalent to the force $P\left(P_{1}, P_{2}, o\right)$ and the moment $M(o, o, o)$. For $x_{3}=o$ we have the conditions

$$
\begin{gathered}
\int_{\Sigma} t_{3 \alpha} \mathrm{d} \sigma=-P_{\alpha}, \\
\int_{\Sigma} t_{33} \mathrm{~d} \sigma=0, \\
\int_{\Sigma}\left(x_{\alpha} t_{33}-\epsilon_{3 \alpha \beta} m_{3 \beta}\right) \mathrm{d} \sigma=0, \\
\int_{\Sigma}\left(\epsilon_{3 \alpha \beta} x_{\alpha} t_{3 \beta}+m_{33}\right) \mathrm{d} \sigma=0 .
\end{gathered}
$$

We seek the solution of the problem (2.1)-(2.3), (2.5), (2.6) and (5.1)-(5.4) in the form 


$$
\begin{aligned}
& u_{\alpha}=-\frac{1}{6} b_{\alpha} x_{3}^{3}+x_{3} \sum_{s=1}^{3} b_{s} u_{\alpha}^{(s)}+\epsilon_{3 \beta \alpha} \tau x_{\beta} x_{3}, \\
& u_{3}=\frac{1}{2}\left(b_{1} x_{1}+b_{2} x_{2}+b_{3}\right) x_{3}^{2}+\tau \Phi+\Psi\left(x_{1}, x_{2}\right), \\
& \varphi_{\alpha}=\frac{1}{2} \epsilon_{3 \alpha \beta} b_{\beta} x_{3}^{2}+\tau \Phi_{\alpha}+\Psi_{\alpha}\left(x_{1}, x_{2}\right), \\
& \varphi_{3}=x_{3} \sum_{s=1}^{3} b_{s} \varphi_{3}^{(s)}+\tau x_{3},
\end{aligned}
$$

where $\Phi, \Phi_{1}, \Phi_{2}$ are the torsion functions considered in Section $4, u_{\alpha}^{(s)}, \varphi_{3}^{(s)}$ are the solutions of the problems $P^{(s)}$ already considered in Section $3, \Psi, \Psi_{1}, \Psi_{2}$ are unknown functions and $b_{i}, \tau$ are unknown constants. From (5.5), (2.3) and (2.2) we obtain

$$
\begin{aligned}
& t_{\alpha \beta}=x_{3} \sum_{s=1}^{3} b_{s} t_{\alpha \beta}^{(s)}+\lambda^{(\rho)} x_{3}\left(b_{1} x_{1}+b_{2} x_{2}+b_{3}\right) \delta_{\alpha \beta}, \\
& t_{33}=\left(\lambda^{(\rho)}+2 \mu^{(\rho)}+\kappa^{(\rho)}\right)\left(b_{1} x_{1}+b_{2} x_{2}+b_{3}\right) x_{3}+\lambda^{(\rho)} x_{3} \sum_{s=1}^{3} b_{s} e_{\alpha \alpha}^{(s)}, \\
& t_{\alpha 3}=\tau\left(T_{\alpha} \Lambda+\mu^{(\rho)} \epsilon_{3 \beta \alpha} x_{\beta}\right)+T_{\alpha} \Omega+\mu^{(\rho)} \sum_{s=1}^{3} b_{s} u_{\alpha}^{(s)}, \\
& t_{3 \alpha}=\tau\left[S_{\alpha} \Lambda+\epsilon_{3 \beta \alpha}\left(\mu^{(\rho)}+\kappa^{(\rho)}\right) x_{\beta}\right]+S_{\alpha} \Omega+\left(\mu^{(\rho)}+\kappa^{(\rho)}\right) \sum_{s=1}^{3} b_{s} u_{\alpha}^{(s)}, \\
& m_{\eta \nu}=\tau\left(M_{\eta \nu} \Lambda+\alpha^{(\rho)} \delta_{\eta \nu}\right)+M_{\eta \nu} \Omega+\alpha^{(\rho)} \delta_{\eta \nu} \sum_{s=1}^{3} b_{s} \varphi 3^{(s)}, \\
& m_{\alpha 3}=\beta^{(\rho)} \epsilon_{3 \alpha \beta} b_{\beta} x_{3}+x_{3} \sum_{s=1}^{3} b_{s} m_{\alpha\}}^{(s)}, \\
& m_{3 \alpha}=x_{3} \sum_{s=1}^{3} b_{s} m_{3 \alpha}^{(s)}+\gamma^{(\rho)} \epsilon_{3 \alpha \beta} b_{\beta} x_{3}, \\
& m_{33}=\left(\alpha^{(\rho)}+\beta^{(\rho)}+\gamma^{(\rho)}\right)\left(\tau+\sum_{s=1}^{3} b_{s} \varphi 3^{(s)}\right)+\alpha^{(\rho)}\left(\tau \Phi_{\alpha, \alpha}+\Psi_{\alpha, \alpha}\right),
\end{aligned}
$$

where $\Omega=\left(\Psi, \Psi_{1}, \Psi_{2}\right)$.

Taking into account the relations $(2.7)-(2.11)$ and $(4.9)-(4.12)$ it follows that the equilibrium eqns (2.1) and the conditions (2.5) and (2.6) reduce to

$$
\begin{gathered}
\mathscr{L}^{(\rho)} \Omega=-\sum_{s=1}^{3} b_{s}\left[\lambda^{(\rho)} e_{\alpha \alpha}^{(s)}+\left(\mu^{(\rho)} u_{\alpha}^{(s)}\right)_{, \alpha}\right]-\left(\lambda^{(\rho)}+2 \mu^{(\rho)}+\kappa^{(\rho)}\right)\left(b_{1} x_{1}+b_{2} x_{2}+b_{3}\right), \\
\mathscr{L}_{\nu}^{(\rho)} \Omega=-\gamma^{(\rho)} \epsilon_{3 \nu \beta} b_{\beta}-\sum_{s=1}^{3} b_{s}\left[\left(\alpha^{(\rho)} \varphi_{3}^{(s)}\right)_{, \nu}+m_{3 \nu}^{(s)}-\epsilon_{\nu \beta 3} \kappa^{(\rho)} u_{\beta}^{(s)}\right], \text { in } \Sigma_{\rho}, \\
{[\Psi]_{1}=[\Psi]_{2},\left[\Psi_{\alpha}\right]_{1}=\left[\Psi_{\alpha}\right]_{2},} \\
{\left[\mathscr{B}^{(1)} \Omega\right](\nu)-\left[\mathscr{B}^{(2)} \Omega\right](\nu)=\left(\mu^{(2)}-\mu^{(1)}\right) \nu_{\alpha} \sum_{s=1}^{3} b_{s} u_{\alpha}^{(s)},} \\
{\left[\mathscr{B}_{\eta}^{(1)} \Omega\right](\nu)-\left[\mathscr{B}_{\eta}^{(2)} \Omega\right](\nu)=\left(\alpha^{(2)}-\alpha^{(1)}\right) \nu_{\eta} \sum_{s=1}^{3} b_{s} \varphi_{3}^{(s)}, \text { on } \Gamma,} \\
\mathscr{B}^{(\rho)} \Omega=-\mu^{(\rho)} n_{\alpha} \sum_{s=1}^{3} b_{s} u_{\alpha}^{(s)}, \mathscr{B}_{\nu}^{(\rho)} \Omega=-\alpha^{(\rho)} n_{\nu} \sum_{s=1}^{3} b_{s} \varphi_{3}^{(s)}, \text { on } L_{\rho}
\end{gathered}
$$

The necessary and sufficient conditions (4.13) for the existence of the solution of the 
boundary value problem (5.7)-(5.9) become

$$
A_{3 s} b_{s}=0
$$

where $A_{3 s}$ are given by (3.8).

Let us consider the conditions (5.1). As in the case of homogeneous cylinders we can prove the relation

$$
\int_{\Sigma} t_{3 \alpha} \mathrm{d} \sigma=\int_{\Sigma}\left(x_{\alpha} t_{33}-\epsilon_{3 \alpha \beta} m_{3 \beta}\right)_{, 3} \mathrm{~d} \sigma
$$

Thus, taking into account (5.6), the conditions (5.1) reduce to

$$
A_{\alpha s} b_{s}=-P_{\alpha}
$$

The system (5.10) and (5.11) determines the constants $b_{i}$. The condition (5.10) is satisfied so that in what follows we assume the function $\Psi, \Psi_{\alpha}$ to be known.

From (5.6) and (5.4) we obtain

$$
\begin{aligned}
\tau D= & -\sum_{\rho=1}^{2} \int_{\Sigma_{\rho}}\left\{\epsilon_{3 \alpha \beta} x_{\alpha}\left[\mu^{(\rho)} \Psi_{, \beta}+\epsilon_{\nu \beta 3} \kappa^{(\rho)} \Psi_{\nu}+\left(\mu^{(\rho)}+\kappa^{(\rho)}\right) \sum_{s=1}^{3} b_{s} u_{\beta}^{(s)}\right]\right. \\
& \left.+\left(\alpha^{(\rho)}+\beta^{(\rho)}+\gamma^{(\rho)}\right) \sum_{s=1}^{3} b_{s} \varphi_{3}^{(s)}+\alpha^{(\rho)} \Psi_{\alpha, \alpha}\right\} \mathrm{d} \sigma
\end{aligned}
$$

where $D$ is given by (4.15). The relation (5.12) determines the constant $\tau$.

The conditions (5.2) and (5.3) are identically satisfied on the basis of the relation (5.6). In this way the problem is reduced to solving plane problems.

\section{TORSION OF A COMPOSITE CIRCULAR CYLINDER}

In what follows we use the method given in Sections $2-4$ in order to study the deformation of a composite circular cylinder. We assume that the domain $\Sigma_{1}$ is bounded by two concentric circles $L$ and $\Gamma$ on radius $R_{1}$ and $r_{2}$, respectively, where $r_{2}<r_{1}$. The domain $\Sigma_{2}$ is bounded by the circle $\Gamma$. Axis $O x_{3}$ of the coordinate system is taken along the axis of the cylinder.

We consider the case when the domains $\Sigma_{1}$ and $\Sigma_{2}$ are occupied by two different homogeneous and isotropic micropolar elastic materials.

In what follows we will use the cylindrical coordinate system $(r, \theta, z)$ and denote by $\bar{e}_{r}, \bar{e}_{\theta}, \bar{e}_{z}$ the unit base vectors of this system.

Let us denote

$$
\bar{u}=u_{r} \bar{e}_{r}+u_{\theta} \bar{e}_{\theta}+u_{z} \bar{e}_{z}, \bar{\varphi}=\varphi_{1} \bar{e}_{r}+\varphi_{\theta} \bar{e}_{\theta}+\varphi_{z} \bar{e}_{2}
$$

Let $\hat{\Phi}=\left(\Phi, \Phi_{r}, \Phi_{\theta}\right)$ be an ordered triplet of functions $\Phi, \Phi_{r}, \Phi_{\theta}$. The eqns (4.10) can be written in the form

$$
L_{z}^{(\rho)} \Phi=0, M_{r}^{(\rho)} \hat{\Phi}=\frac{1}{2}\left(s^{(\rho)}\right)^{2} r^{3}, M_{\ell}^{(\rho)} \hat{\Phi}=0, \quad \text { in } \quad \Sigma_{\rho}
$$

where

$$
\begin{aligned}
L_{z}^{(\rho)} \hat{\Phi} & =\left(D_{r}^{2}+D_{\theta}^{2}\right) \Phi+e^{(\rho)} r\left(D_{r}+1\right) \Phi_{\theta}-e^{(\rho)} r D_{\theta} \Phi_{r} \\
M_{r}^{(\rho)} \hat{\Phi} & =\left(D_{r}^{2}+b^{(\rho)} D_{\theta}^{2}-\left(s^{(\rho)}\right)^{2} r^{2}-1\right) \Phi_{r}+\left[\left(1-b^{(\rho)}\right) D_{r}-\left(1+b^{(\rho)}\right)\right] D_{\theta} \Phi_{\theta}+\frac{1}{2}\left(s^{(\rho)}\right)^{2} r D_{\theta} \Phi
\end{aligned}
$$




$$
\begin{aligned}
M_{f}^{(\rho)} \hat{\Phi} & =\left[b^{(\rho)}\left(D_{r}^{2}-1\right)-r^{2}\left(s^{(\rho)}\right)^{2}+D_{\theta}^{2}\right] \Phi_{\theta}+\left[\left(1-b^{(\rho)}\right) D_{r}+\left(1+b^{(\rho)}\right)\right] D_{\theta} \Phi_{r}-\frac{1}{2} r\left(s^{(\rho)}\right)^{2} D_{r} \Phi, \\
D_{r} & =r \frac{\mathrm{d}}{\mathrm{d} r}, D_{\theta}=\frac{\mathrm{d}}{\mathrm{d} \theta}, \\
e^{(\rho)} & =\frac{\kappa^{(\rho)}}{\mu^{(\rho)}+\kappa^{(\rho)},}\left(s^{(\rho)}\right)^{2}=\frac{2 \kappa^{(\rho)}}{\alpha^{(\rho)}+\beta^{(\rho)}+\gamma^{(\rho)},} b^{(\rho)}=\frac{\gamma^{(\rho)}}{\alpha^{(\rho)}+\beta^{(\rho)}+\gamma^{(\rho)}} .
\end{aligned}
$$

From the conditions (4.11) and (4.12) it follows

$$
\begin{aligned}
& {[\hat{\Phi}]_{1}=[\dot{\Phi}]_{2}, T_{z}^{(1)} \hat{\Phi}-T_{z}^{(2)} \dot{\Phi}=0,} \\
& S_{r}^{(1)} \hat{\Phi}-S_{r}^{(2)} \hat{\Phi}=\alpha^{(2)}-\alpha^{(1)}, S_{\theta}^{(1)} \hat{\Phi}-S_{\theta}^{(2)} \hat{\Phi}=0 \text {, on } \Gamma \text {, } \\
& T_{z}^{(1)} \Phi=0, S_{r}^{(1)} \hat{\Phi}=-\alpha^{(1)}, S_{\theta}^{(1)} \hat{\Phi}=0, \quad \text { on } L,
\end{aligned}
$$

where

$$
\begin{aligned}
T_{z}^{(\rho)} \hat{\Phi}= & \frac{1}{r}\left(\mu^{(\rho)}+\kappa^{(\rho)}\right) D_{r} \Phi+\kappa^{(\rho)} \Phi_{\theta}, r S S^{(\rho)} \hat{\Phi}=\gamma^{(\rho)} D_{r} \Phi_{\theta} \\
& +\beta^{(\rho)}\left(D_{\theta} \Phi_{r}-\Phi_{\theta}\right) \\
r S_{r}^{(\rho)} \hat{\Phi}= & \left(\alpha^{(\rho)}+\beta^{(\rho)}+\gamma^{(\rho)}\right) D_{r} \Phi_{r}+\alpha^{(\rho)}\left(D_{\theta} \Phi_{\theta}+\Phi_{r}\right) .
\end{aligned}
$$

Let us determine the solution of the problem (6.1) and (6.2). We seek this solution as function only of $r$. It is easy to verify that a particular solution of (6.1) is given by

$$
\tilde{\Phi}=0, \tilde{\Phi}_{r}=-\frac{1}{2} r, \tilde{\Phi}_{\theta}=0
$$

The homogeneous solution of (6.1) can be obtained as in [12]. We obtain

$$
\begin{aligned}
& \Phi^{o}=-e^{(2)} A_{1} \int I_{1}\left(\delta^{(2)} r\right) \mathrm{d} r+A_{2}, \\
& \Phi_{r}^{o}=A_{3} I_{1}\left(\left(^{(2)} r\right), \Phi_{\theta}^{o}=A_{1} I_{1}\left(\delta^{(2)} r\right), \text { for } 0 \leq r \leq r_{2},\right. \\
& \Phi^{o}=-e^{(1)} \int\left[B_{1} I_{1}\left(\delta^{(1)} r\right)+B_{4} K_{1}\left(\delta^{(1)} r\right)\right] \mathrm{d} r+B_{5}\left[1+\frac{e^{(1)} \kappa^{(1)}}{\gamma^{(1)} \delta^{(1)}}\right] \ln r+B_{2}, \\
& \Phi_{r}^{o}=B_{3} I_{1}\left(\left(^{(1)} r\right)+B_{6} K_{1}\left(s^{(1)} r\right),\right. \\
& \Phi_{\theta}^{o}=B_{1} I_{1}\left(\delta^{(1)} r\right)+B_{4} K_{1}\left(\delta^{(1)} r\right)-\frac{\kappa^{(1)}}{\gamma^{(1)} \delta^{(1) 2}} \cdot \frac{1}{r} B_{5}, \text { for } r_{2} \leq r \leq r_{1},
\end{aligned}
$$

where $A_{i}, B_{i}, B_{i+3}(i=1,2,3)$ are unknown constants, $I_{n}(x), K_{n}(x)$ are the modified Bessel functions of order $n$, and

$$
\left(\delta^{(\rho)}\right)^{2}=\left(2-e^{(\rho)}\right) \sigma^{(\rho)}, \sigma^{(\rho)}=\frac{\kappa^{(\rho)}}{\gamma^{(\rho)}}
$$

By substituting (6.3) and (6.4) in the conditions (6.2) we obtain for the unknown constants the following values

$$
\begin{aligned}
& A_{1}=B_{1}=B_{4}=B_{5}=0, A_{2}=B_{2}, \\
& B_{3}=\left(Q_{5} Q_{7}-Q_{4} Q_{8}\right) /\left(Q_{3} Q_{7}-Q_{4} Q_{6}\right), \\
& B_{6}=\left(Q_{3} Q_{8}-Q_{5} Q_{6}\right) /\left(Q_{3} Q_{7}-Q_{4} Q_{6}\right), \\
& A_{3}=Q_{1} B_{3}+Q_{2} B_{6},
\end{aligned}
$$


where

$$
\begin{aligned}
Q_{1}= & I_{1}\left(s^{(1)} r_{2}\right) / I_{1}\left(s^{(2)} r_{2}\right), Q_{2}=K_{1}\left(s^{(1)} r_{2}\right) / I_{1}\left(s^{(2)} r_{2}\right), \\
Q_{3}= & Q_{1}\left[\left(\alpha^{(2)}+\beta^{(2)}+\gamma^{(2)}\right) I_{1}^{\prime}\left(s^{(2)} r_{2}\right)+\alpha^{(2)} I_{1}\left(s^{(2)} r_{2}\right) /\left(s^{(2)} r_{2}\right)\right] \\
& -\left[\left(\alpha^{(1)}+\beta^{(1)}+\gamma^{(1)}\right) I_{1}\left(s^{(1)} r_{2}\right)+\alpha^{(1)} I_{1}\left(s^{(1)} r_{2}\right) /\left(s^{(1)} r_{2}\right)\right] s^{(1)} / s^{(2)}, \\
Q_{4}= & Q_{2}\left[\left(\alpha^{(2)}+\beta^{(2)}+\gamma^{(2)}\right) I_{1}\left(s^{(2)} r_{2}\right)+\alpha^{(2)} I_{1}\left(s^{(2)} r_{2}\right) /\left(s^{(2)} r_{2}\right)\right] \\
& -\left[\left(\alpha^{(1)}+\beta^{(1)}+\gamma^{(1)}\right) K_{1}^{\prime}\left(s^{(1)} r_{2}\right)+\alpha^{(1)} K_{1}\left(s^{(1)} r_{2}\right) /\left(s^{(1)} r_{2}\right)\right] s^{(1)} / s^{(2)}, \\
Q_{5}= & {\left.\left[\left(\beta^{(2)}+\gamma^{(2)}\right)-\left(\beta^{(1)}+\gamma^{(1)}\right)\right] /\left(2 s^{(2)}\right), Q_{6}=\left(\alpha^{(1)}+\beta^{(1)}+\gamma^{(1)}\right) I_{1}\left(s^{(1)} r_{1}\right)+\alpha^{(1)} r_{1}\right) } \\
& +\alpha^{(1)} I_{1}\left(s^{(1)} r_{1}\right) /\left(s^{(1)} r_{1}\right), \\
Q_{7}= & \left(\alpha^{(1)}+\beta^{(1)}+\gamma^{(1)}\right) K_{1}^{\prime}\left(s^{(1)} r_{1}\right)+\alpha^{(1)} K_{1}\left(s^{(1)} r_{1}\right) /\left(s^{(1)} r_{1}\right), Q_{8}=\left(\beta^{(1)}+\gamma^{(1)}\right) /\left(2 s^{(1)}\right), \\
I_{1}(r)= & \frac{\mathrm{d} I_{1}}{\mathrm{~d} r}, K_{1}(r)=\frac{\mathrm{d} K_{1}}{\mathrm{~d} r} .
\end{aligned}
$$

The solution of problem (6.1), (6.2) has the form

$$
\begin{gathered}
\Phi=A_{2}, \Phi_{r}=-\frac{1}{2} r+A_{3} I_{1}\left(s^{(2)} r\right), \Phi_{\theta}=0, \text { for } 0 \leq r \leq r_{2}, \\
\Phi=A_{2}, \Phi_{r}=-\frac{1}{2} r+B_{3} I_{1}\left(s^{(1)} r\right)+B_{6} K_{1}\left(s^{(1)} r\right), \Phi_{\theta}=0, \text { for } r_{2} \leq r \leq r_{1} .
\end{gathered}
$$

Therefore, the solution of the torsion problem for the composite circular cylinder is

where

$$
\begin{aligned}
& u_{r}=0, u_{\theta}=\tau r z, u_{z}=\tau \Phi, \\
& \varphi_{r}=\tau \Phi_{n} \varphi_{\theta}=\tau \Phi_{\theta}, \varphi_{z}=\tau z,
\end{aligned}
$$

$$
\begin{aligned}
\tau= & -M_{3} / D, \\
D= & \frac{1}{4}\left(2 \mu^{(2)}+\kappa^{(2)}\right) \pi r_{2}^{4}+\left(\beta^{(2)}+\gamma^{(2)}\right) \pi r_{2}^{2}+\frac{2}{s^{(2)}} \pi \kappa^{(2)} A_{3} r_{2}^{2} I_{2}\left(s^{(2)} r_{2}\right) \\
& +2 \pi \alpha^{(2)} A_{3} r_{2} I_{1}\left(s^{(2)} r_{2}\right)+\frac{1}{4}\left(2 \mu^{(1)}+\kappa^{(1)}\right) \pi\left(r_{1}^{4}-r_{2}^{4}\right)+\left(\beta^{(1)}+\gamma^{(1)}\right) \pi\left(r_{1}^{2}-r_{2}^{2}\right) \\
& +\left\{B_{3}\left[r_{1}^{2} I_{2}\left(s^{(1)} r_{1}\right)-r_{2}^{2} I_{2}\left(s^{(1)} r_{2}\right)\right]-B_{6}\left[r_{1}^{2} K_{2}\left(s^{(1)} r_{1}\right)-r_{2}^{2} K_{2}\left(s^{(1)} r_{2}\right)\right]\right\} 2 \pi \kappa^{(1)} / s^{(1)} \\
& +2 \pi \alpha^{(1)}\left\{B_{3}\left[r_{1} I_{1}\left(s^{(1)} r_{1}\right)-r_{2} I_{1}\left(s^{(1)} r_{2}\right)\right]+B_{6}\left[r_{1} K_{1}\left(s^{(1)} r_{1}\right)-r_{2} K_{1}\left(s^{(1)} r_{2}\right)\right]\right\} .
\end{aligned}
$$

It is easy to see that the constant $A_{2}$ characterizes a rigid translation.

\section{EXTENSION AND BENDING}

Let $U=\left(u_{r}, u_{\theta}, \varphi_{z}\right)$ be an ordered triplet of functions $u_{m} u_{\theta}, \varphi_{z}$. We introduce the notations

$$
\begin{aligned}
L_{r}^{(\rho)} U & =\left(D_{r}^{2}+c^{(\rho)} D_{\theta}^{2}-1\right) u_{r}+\left[\left(1-c^{(\rho)}\right) D_{r}-\left(1+c^{(\rho)}\right)\right] D_{\theta} u_{\theta}+d^{(\rho)} r D_{\theta} \varphi_{z}, \\
L_{\theta}^{(\rho)} U & =\left[\left(1-c^{(\rho)}\right) D_{r}+\left(1+c^{(\rho)}\right)\right] D_{\theta} u_{r}+\left[c^{(\rho)}\left(D_{r}^{2}-1\right)+D_{\theta}^{2}\right] u_{\theta}-d^{(\rho)} r D_{r} \varphi_{z}, \\
M_{z}^{(\rho)} U & =-\sigma^{(\rho)} r D_{\theta} u_{r}+\sigma^{(\rho)} r\left(D_{r}+1\right) u_{\theta}+\left(D_{r}^{2}+D_{\theta}^{2}-2 \sigma^{(\rho)} r^{2}\right) \varphi_{z}, \\
T_{r}^{(\rho)} U & =\left[\left(\lambda^{(\rho)}+2 \mu^{(\rho)}+\kappa^{(\rho)}\right) D_{r} u_{r}+\lambda^{(\rho)}\left(D_{\theta} u_{\theta}+u_{r}\right)\right] / r, \\
T \gamma^{(\rho)} U & =\frac{1}{r}\left[\left(\mu^{(\rho)}+\kappa^{(\rho)}\right) D_{r} u_{\theta}+\mu^{(\rho)}\left(D_{\theta} u_{r}-u_{\theta}\right)\right]-\kappa^{(\rho)} \varphi_{z}, \\
S_{z}^{(\rho)} U & =\frac{1}{r} \gamma^{(\rho)} D_{r} \varphi_{z},
\end{aligned}
$$


where

$$
c^{(\rho)}=\frac{\mu^{(\rho)}+\kappa^{(\rho)}}{\lambda^{(\rho)}+2 \mu^{(\rho)}+\kappa^{(\rho)}}, d^{(\rho)}=\frac{\kappa^{(\rho)}}{\lambda^{(\rho)}+2 \mu^{(\rho)}+\kappa^{(\rho)}}
$$

Using these notations, the problems $P^{(s)}$ can be written in the form

$$
L_{r}^{(\rho)} U^{(1)}=-\left(1-2 c^{(\rho)}+d^{(\rho)}\right) r^{2} \cos \theta, L_{\theta}^{(\rho)} U^{(1)}=\left(1-2 c^{(\rho)}+d^{(\rho)}\right) r^{2} \sin \theta, M_{z}^{(\rho)} U^{(1)}=0, \text { in } \quad \Sigma_{\rho r}
$$

$$
\begin{aligned}
& {\left[U^{(1)}\right]_{1}=\left[U^{(1)}\right]_{2}, T_{r}^{(2)} U^{(1)}-T_{r}^{(1)} U^{(1)}=\left(\lambda^{(1)}-\lambda^{(2)}\right) r_{2} \cos \theta,} \\
& T_{\theta}^{(2)} U^{(1)}-T_{\theta}^{(1)} U^{(1)}=0, S_{z}^{(2)} U^{(1)}-S_{z}^{(1)} U^{(1)}=\left(\beta^{(2)}-\beta^{(1)}\right) \sin \theta, \text { on } \Gamma, \\
& T_{r}^{(1)} U^{(1)}=-\lambda^{(1)} r_{1} \cos \theta, T_{\theta}^{(1)} U^{(1)}=0, S_{z}^{(1)} U^{(1)}=\beta^{(1)} \sin \theta, \text { on } L,
\end{aligned}
$$

$L_{r}^{(\rho)} U^{(2)}=-\left(1-2 c^{(\rho)}+d^{(\rho)}\right) r^{2} \sin \theta, L_{\theta}^{(\rho)} U^{(2)}=-\left(1-2 c^{(\rho)}+d^{(\rho)}\right) r^{2} \cos \theta, M_{z}^{(\rho)} U^{(2)}=0$, in $\quad \Sigma_{\rho,}$

$$
\begin{aligned}
& {\left[U^{(2)}\right]_{1}=\left[U^{(2)}\right]_{2}, T_{r}^{(2)} U^{(2)}-T_{r}^{(1)} U^{(2)}=\left(\lambda^{(1)}-\lambda^{(2)}\right) r_{2} \sin \theta,} \\
& T_{\theta}^{(2)} U^{(2)}-T_{\theta}^{(1)} U^{(2)}=0, S_{z}^{(2)} U^{(2)}-S_{z}^{(1)} U^{(2)}=\left(\beta^{(1)}-\beta^{(2)}\right) \cos \theta, \text { on } \Gamma, \\
& T_{r}^{(1)} U^{(2)}=-\lambda^{(1)} r_{1} \sin \theta, T_{\theta}^{(1)} U^{(2)}=0, S_{z}^{(1)} U^{(2)}=-\beta^{(1)} \cos \theta, \text { on } L, \\
& L_{r}^{(\rho)} U^{(3)}=0, L_{\theta}^{(\rho)} U^{(3)}=0, M_{z}^{(\rho)} U^{(3)}=0, \text { in } \Sigma_{\rho}, \\
& {\left[U^{(3)}\right]_{1}=\left[U^{(3)}\right]_{2}, T_{r}^{(2)} U^{(3)}-T_{r}^{(1)} U^{(3)}=\lambda^{(1)}-\lambda^{(2)},} \\
& T_{\theta}^{(2)} U^{(3)}-T_{\theta}^{(1)} U^{(3)}=0, S_{z}^{(2)} U^{(3)}-S_{z}^{(1)} U^{(3)}=0, \text { on } \Gamma, \\
& T_{r}^{(1)} U^{(3)}=-\lambda^{(1)}, T_{\theta}^{(1)} U^{(3)}=0, S_{z}^{(1)} U^{(3)}=0, \text { on } L,
\end{aligned}
$$

where $U^{(s)}=\left(u_{r}^{(s)}, u_{\theta}^{(s)}, \varphi_{2}^{(s)}\right)$.

Let us determine the solutions of the problems $P^{(s)}$. First we consider the problems $P^{(\beta)}(\beta=1,2)$. We seek the solutions of these problems in the form

$$
U^{(\beta)}=\left(A^{(\beta)} \cos \theta+A_{2}^{(\beta)} \sin \theta, B^{(\beta)} \cos \theta+B_{2}^{(\beta)} \sin \theta, C^{(\beta)} \cos \theta+C_{2}^{(\beta)} \sin \theta\right),
$$

where $A_{\alpha}^{(\beta)}, B_{\alpha}^{(\beta)}, C_{\alpha}^{(\beta)}$ are functions of $r$. From (7.3)-(7.6) we obtain

$$
\begin{aligned}
& A_{2}^{(1)}=B_{1}^{(1)}=C_{1}^{(1)}=A_{1}^{(2)}=B_{2}^{(2)}=C_{2}^{(2)}=0, \\
& A_{1}^{(1)}=A_{2}^{(2)}=v_{n} B_{2}^{(1)}=-B_{1}^{(2)}=v_{\theta}, C_{2}^{(1)}=-C_{1}^{(2)}=\psi_{2},
\end{aligned}
$$

where $v_{n} v_{\theta}, \psi_{z}$ satisfy the following equations

$$
\begin{aligned}
& \left(D_{r}^{2}-1-c^{(\rho)}\right) v_{r}+\left[\left(1-c^{(\rho)}\right) D_{r}-\left(1+c^{(\rho)}\right)\right] v_{\theta}+d^{(\rho)} r \psi_{z}=-\left(1+d^{(\rho)}-2 c^{(\rho)}\right) r^{2}, \\
& {\left[\left(1-c^{(\rho)}\right) D_{r}+\left(1+c^{(\rho)}\right)\right] v_{r}-\left[c^{(\rho)}\left(D_{r}^{2}-1\right)-1\right] v_{\theta}+d^{(\rho)} r D_{r} \psi_{z}=-\left(1+d^{(\rho)}-2 c^{(\rho)}\right) r^{2},} \\
& \left(D_{r}^{2}-1-2 \sigma^{(\rho)} r^{2}\right) \psi_{z}+\sigma^{(\rho)} r\left(D_{r}+1\right) v_{\theta}+\sigma^{(\rho)} v_{r}=0, \text { in } \Sigma_{p},
\end{aligned}
$$

and the conditions

$$
\begin{aligned}
& {[W]_{1}=[W]_{2}, \mathscr{T}_{r}^{(2)} W-\mathscr{T}_{r}^{(1)} W=\left(\lambda^{(1)}-\lambda^{(2)}\right) r_{2},} \\
& \mathscr{F}_{\theta}^{(2)} W-\mathscr{T}_{\theta}^{(1)} W=0, \mathscr{F}_{z}^{(2)} W-\mathscr{T}_{z}^{(1)} W=\beta^{(2)}-\beta^{(1)}, \quad \text { on } \quad r=r_{2}, \\
& \mathscr{F}_{r}^{(1)} W=-\lambda^{(1)} r_{1}, \mathscr{T}_{\theta}^{(1)} W=0, \mathscr{T}_{z}^{(1)} W=\beta^{(1)}, \quad \text { on } \quad r=r_{1} .
\end{aligned}
$$

In the above relations we have used the notations 


$$
\begin{aligned}
W & =\left(v_{r}, v_{\theta}, \psi_{z}\right), \\
\mathscr{T}_{r}^{(\rho)} W & =\left[\left(\lambda^{(\rho)}+2 \mu^{(\rho)}+\kappa^{(\rho)}\right) D_{r} v_{r}+\lambda^{(\rho)}\left(v_{r}+v_{\theta}\right)\right] / r, \\
\mathscr{T}_{g}^{(\rho)} W & =\left[\left(\mu^{(\rho)}+\kappa^{(\rho)}\right) D_{r} v_{\theta}-\mu^{(\rho)}\left(v_{r}+v_{\theta}\right)-r \kappa^{(\rho)} \psi_{z}\right] / r, \mathscr{T}_{z}^{(\rho)} W=\frac{\gamma^{(\rho)}}{r} D_{r} \psi_{z} .
\end{aligned}
$$

A particular solution of (7.11) is given by

$$
\bar{v}_{r}=-\frac{1}{8} r^{2}, \bar{v}_{\theta}=-\frac{5}{8} r^{2}, \tilde{\psi}_{2}=-r .
$$

The homogeneous solutions of (7.11) can be derived from the general solutions given in [12]. We find

$$
\begin{aligned}
v_{r}^{o}= & C_{1}+\left(2-6 c^{(2)}+3 d^{(2)}\right) C_{2} r^{2}+\frac{e^{(2)}}{2 \delta^{(2)}} C_{3}\left[I_{2}\left(\delta^{(2)} r\right)-I_{0}\left(\delta^{(2)} r\right)\right], \\
v_{\theta}^{o}= & -C_{1}-\left(6-2 c^{(2)}+d^{(2)}\right) C_{2} r^{2}+\frac{e^{(2)}}{\delta^{(2)}} C_{3} I_{1}^{\prime}\left(\delta^{(2)} r\right), \\
\psi_{z}^{o}= & -8 C_{2} r+C_{3} I_{1}\left(\delta^{(2)} r\right), \text { for } 0 \leq r \leq r_{2}, \\
v_{r}^{o}= & D_{1}+\left(2-6 c^{(1)}+3 d^{(1)}\right) D_{2} r^{2}+\frac{e^{(1)}}{2 \delta^{(1)}}\left\{D_{3}\left[I_{2}\left(\delta^{(1)} r\right)-I_{o}\left(\delta^{(1)} r\right)\right]\right. \\
& \left.-D_{4}\left[K_{2}\left(\delta^{(1)} r\right)-K_{0}\left(\delta^{(1)} r\right)\right]\right\}+D_{5} r^{-2}-\frac{1}{2} d^{(1)} D_{6}+c^{(1)}\left(2+2 c^{(1)}-d^{(1)}\right) D_{6} \ln r, \\
v_{\theta}^{o}= & -D_{1}-\left(6-2 c^{(1)}+d^{(1)}\right) D_{2} r^{2}+\frac{e^{(1)}}{\delta^{(1)}}\left[D_{3} I_{1}^{\prime}\left(\delta^{(1)} r\right)+D_{4} K_{1}^{\prime}\left(\delta^{(1)} r\right)\right] \\
& +D_{5} r^{-2}-\frac{1}{2}\left[2\left(1-c^{(1)}\right)\left(2 c^{(1)}-d^{(1)}\right)+d^{(1)}\right] D_{6}-c^{(1)}\left(2+2 c^{(1)}-d^{(1)}\right) D_{6} \ln r, \\
\psi_{2}^{o}= & -8 D_{2} r+D_{3} I_{1}\left(\delta^{(1)} r\right)+D_{4} K_{1}\left(\delta^{(1)} r\right)-2 c^{(1)} D_{6} r^{-1}, \text { for } r_{2} \leq r \leq r_{1},
\end{aligned}
$$

where $C_{i}, D_{i}, D_{i+3}(i=1,2,3)$ are unknown constants.

Substituting these values in (7.12) we find

$$
\begin{aligned}
D_{1}= & C_{1}+2 r_{2}^{2}\left[\left(2-2 c^{(2)}+d^{(2)}\right) C_{2}-\left(2-2 c^{(1)}+d^{(1)}\right) D_{2}\right]-\frac{e^{(2)}}{2 \delta^{(2)}} C_{3} I_{o}\left(\delta^{(2)} r_{2}\right) \\
& +\frac{e^{(1)}}{2 \delta^{(1)}}\left[D_{3} I_{o}\left(\delta^{(1)} r_{2}\right)-D_{4} K_{o}\left(\delta^{(1)} r_{2}\right)\right], \\
D_{2}= & H_{1}+H_{2} D_{4}+H_{3} D_{5}, D_{3}=H_{4}+H_{5} D_{4}+H_{6} D_{5}, \\
D_{4}= & \left(H_{10} H_{11}-H_{8} H_{12}\right) /\left(H_{7} H_{10}-H_{8} H_{9}\right), D_{5}=\left(H_{7} H_{12}-H_{9} H_{11}\right) /\left(H_{7} H_{10}-H_{8} H_{9}\right), \\
D_{6}= & 0 \\
C_{2}= & H_{13}+H_{14} D_{2}+H_{15} D_{3}+H_{16} D_{4}+H_{17} D_{5}, \\
C_{3}= & H_{18}+H_{19} D_{2}+H_{20} D_{3}+H_{21} D_{4}+H_{22} D_{5},
\end{aligned}
$$

where

$$
\begin{aligned}
& H_{1}=\left[-\frac{1}{4} \delta^{(1)} r_{1}^{2} I_{1}^{\prime}\left(\delta^{(1)} r_{1}\right)-\frac{e^{(1)}}{\delta^{(1)}} \cdot \frac{\beta^{(1)}+\gamma^{(1)}}{\gamma^{(1)}} I_{2}\left(\delta^{(1)} r_{1}\right)\right] / \Delta_{1}, \\
& H_{2}=e^{(1)}\left[I_{1}^{\prime}\left(\delta^{(1)} r_{1}\right) K_{2}\left(\delta^{(1)} r_{1}\right)+K_{1}^{\prime}\left(\delta^{(1)} r_{1}\right) I_{2}\left(\delta^{(1)} r_{1}\right)\right] / \Delta_{1}, \\
& H_{3}=-2 \delta^{(1)} I_{1}^{\prime}\left(\delta^{(1)} r_{1}\right) /\left(r_{1}^{2} \Delta_{1}\right), H_{4}=2 r_{1}^{2}\left[\frac{\beta^{(1)}+\gamma^{(1)}}{\gamma^{(1)}}\left(2-2 c^{(1)}+d^{(1)}\right)-1\right] / \Delta_{1},
\end{aligned}
$$




$$
\begin{aligned}
H_{5}= & {\left[-2\left(2-2 c^{(1)}+d^{(1)}\right) \delta^{(1)} r_{1}^{2} K_{1}^{\prime}\left(\delta^{(1)} r_{1}\right)+\frac{8 e^{(1)}}{\delta^{(1)}} K_{2}\left(\delta^{(1)} r_{1}\right)\right] / \Delta_{1}, } \\
H_{6}= & -16 /\left(r_{1}^{2} \Delta_{1}\right) \\
\Delta_{1}= & 8 \frac{e^{(1)}}{\delta^{(1)}} I_{2}\left(\delta^{(1)} r_{1}\right)+2\left(2-2 c^{(1)}+d^{(1)}\right) \delta^{(1)} r_{1}^{2} I_{1}\left(\delta^{(1)} r_{1}\right) ; \\
H_{7}= & \frac{e^{(1)}}{\delta^{(1)}}\left[K_{2}\left(\delta^{(1)} r_{2}\right)-H_{5} I_{2}\left(\delta^{(1)} r_{2}\right)\right]+2 r_{2}^{2}\left[\left(2+2 c^{(1)}-d^{(1)}\right) H_{2}-\left(2+2 c^{(2)}-d^{(2)}\right)\right. \\
& \left.\times\left(H_{16}+H_{5} H_{15}+H_{2} H_{14}\right)\right]+\frac{e^{(2)}}{\delta^{(2)}} I_{2}\left(\delta^{(2)} r_{2}\right)\left(H_{21}+H_{5} H_{20}+H_{2} H_{19}\right) \\
H_{8}= & -2 r_{2}^{-2}-\frac{e^{(1)}}{\delta^{(1)}} H_{6} I_{2}\left(\delta^{(1)} r_{2}\right)+2 r_{2}^{2}\left[\left(2+2 c^{(1)}-d^{(1)}\right) H_{3}-\left(2+2 c^{(2)}-d^{(2)}\right)\right. \\
& \left.\times\left(H_{17}+H_{6} H_{15}+H_{3} H_{14}\right)\right]+\frac{e^{(2)}}{\delta^{(2)}} I_{2}\left(\delta^{(2)} r_{2}\right)\left(H_{22}+H_{6} H_{20}+H_{3} H_{19}\right), \\
H_{9}= & -K_{1}\left(\delta^{(1)} r_{2}\right)+8 r_{2} H_{2} \cdots I_{1}\left(\delta^{(1)} r_{2}\right) H_{5}-8 r_{2}\left(H_{16}+H_{5} H_{15}+H_{2} H_{14}\right) \\
& +I_{1}\left(\delta^{(2)} r_{2}\right)\left(H_{21}+H_{5} H_{20}+H_{2} H_{19}\right) \\
H_{10}= & 8 r_{2} H_{3}-I_{1}\left(\delta^{(1)} r_{2}\right) H_{6}-8 r_{2}\left(H_{17}+H_{6} H_{15}+H_{3} H_{14}\right)+I_{1}\left(\delta^{(2)} r_{2}\right)\left(H_{22}+H_{6} H_{20}+H_{3} H_{19}\right), \\
H_{11}= & -2 r_{2}^{2}\left(2+2 c^{(1)}-d^{(1)}\right) H_{1}+\frac{e^{(1)}}{\delta^{(1)}} I_{2}\left(\delta^{(1)} r_{2}\right) H_{4}+2\left(2+2 c^{(2)}-d^{(2)}\right) r_{2}^{2}\left(H_{13}+H_{1} H_{14}+H_{4} H_{15}\right) \\
& -\frac{e^{(2)}}{\delta^{(2)}} I_{2}\left(\delta^{(2)} r_{2}\right)\left(H_{18}+H_{1} H_{19}+H_{4} H_{20}\right),
\end{aligned}
$$$$
H_{12}=-8 r_{2} H_{1}+I_{1}\left(\delta^{(1)} r_{2}\right) H_{4}+8 r_{2}\left(H_{13}+H_{1} H_{14}+H_{4} H_{15}\right)-I_{1}\left(\delta^{(2)} r_{2}\right)\left(H_{18}+H_{1} H_{19}+H_{4} H_{20}\right) \text {, }
$$$$
H_{13}=\left[(\chi-1) \frac{e^{(2)}\left(\beta^{(2)}+\gamma^{(2)}\right)}{\delta^{(2)} \gamma^{(2)}} I_{2}\left(\delta^{(2)} r_{2}\right)+\frac{1}{4}(\omega-1) \delta^{(2)} r_{2}^{2} I_{1}^{\prime}\left(\delta^{(2)} r_{2}\right)\right] / \Delta_{2} \text {, }
$$$$
H_{14}=\left[8 \epsilon \frac{e^{(2)}}{\delta^{(2)}} I_{2}\left(\delta^{(2)} r_{2}\right)+2 \omega\left(2-2 c^{(1)}+d^{(1)}\right) \delta^{(2)} r_{2}^{2} I_{1}^{\prime}\left(\delta^{(2)} r_{2}\right)\right] / \Delta_{2} \text {, }
$$$$
H_{15}=\left[-\epsilon e^{(2)} \frac{\delta^{(1)}}{\delta^{(2)}} I_{2}\left(\delta^{(2)} r_{2}\right) I_{1}^{\prime}\left(\delta^{(1)} r_{2}\right)+\omega e^{(1)} \frac{\delta^{(2)}}{\delta^{(1)}} I_{2}\left(\delta^{(1)} r_{2}\right) I_{1}^{\prime}\left(\delta^{(2)} r_{2}\right)\right] / \Delta_{2},
$$$$
H_{16}=\left[\epsilon e^{(2)} \frac{\delta^{(1)}}{\delta^{(2)}} I_{2}\left(\delta^{(2)} r_{2}\right) K_{1}^{\prime}\left(\delta^{(1)} r_{2}\right)+\omega e^{(1)} \frac{\delta^{(2)}}{\delta^{(1)}} K_{2}\left(\delta^{(1)} r_{2}\right) I_{1}^{\prime}\left(\delta^{(2)} r_{2}\right)\right] / \Delta_{2},
$$$$
H_{17}=2 \omega \delta^{(2)} I_{1}^{\prime}\left(\delta^{(2)} r_{2}\right) /\left(r_{2}^{2} \Delta_{2}\right) \text {, }
$$$$
H_{18}=2 r_{2}^{2}\left[\omega-1+\left(2-2 c^{(2)}+d^{(2)}\right) \frac{\beta^{(2)}+\gamma^{(2)}}{\gamma^{(2)}}(1-\chi)\right] / \Delta_{2},
$$$$
H_{19}=16 r_{2}^{2}\left[\omega\left(2-2 c^{(1)}+d^{(1)}\right)-\epsilon\left(2-2 c^{(2)}+d^{(2)}\right)\right] / \Delta_{2},
$$$$
H_{20}=\left[8 \omega \frac{e^{(1)}}{\delta^{(1)}} I_{2}\left(\delta^{(1)} r_{2}\right)+2 \epsilon\left(2-2 c^{(2)}+d^{(2)}\right) \delta^{(1)} r_{2}^{2} I_{1}^{\prime}\left(\delta^{(1)} r_{2}\right)\right] / \Delta_{2} \text {, }
$$$$
H_{21}=\left[-8 \omega \frac{e^{(1)}}{\delta^{(n)}} K_{2}\left(\delta^{(1)} r_{2}\right)+2 \epsilon\left(2-2 c^{(2)}+d^{(2)}\right) \delta^{(1)} r_{2}^{2} K_{1}^{\prime}\left(\delta^{(1)} r_{2}\right)\right] / \Delta_{2},
$$$$
H_{22}=16 \omega /\left(r_{2}^{2} \Delta_{2}\right) \text {, }
$$

$$
\begin{aligned}
& \Delta_{2}=8 \frac{e^{(2)}}{\delta^{(2)}} I_{2}\left(\delta^{(2)} r_{2}\right)+2 \delta^{(2)} r_{2}^{2}\left(2-2 c^{(2)}+d^{(2)}\right) I_{1}^{\prime}\left(\delta^{(2)} r_{2}\right) ; \\
& \omega=\frac{2 \mu^{(1)}+\kappa^{(1)}}{2 \mu^{(2)}+\kappa^{(2)}}, \epsilon=\frac{\gamma^{(1)}}{\gamma^{(2)}, \chi=\frac{\beta^{(1)}+\gamma^{(1)}}{\beta^{(2)}+\gamma^{(2)}} .}
\end{aligned}
$$

Therefore, the solutions of the problems $P^{(\beta)}$ are 


$$
\begin{gathered}
u_{r}^{(1)}=\left(-\frac{1}{8} r^{2}+v_{r}^{o}\right) \cos \theta, u_{\theta}^{(1)}=\left(-\frac{5}{8} r^{2}+v_{\theta}^{o}\right) \sin \theta, \varphi_{z}^{(1)}=\left(-r+\psi_{z}^{o}\right) \sin \theta, \\
u_{r}^{(2)}=\left(-\frac{1}{8} r^{2}+v_{r}^{o}\right) \sin \theta, u_{\theta}^{(2)}=\left(\frac{5}{8} r^{2}-v_{\theta}^{o}\right) \cos \theta, \varphi_{z}^{(2)}=\left(r-\psi_{z}^{o}\right) \cos \theta,
\end{gathered}
$$

where $v_{r}^{o}, v_{\theta}^{o}, \psi_{z}^{o}$ are given by (7.14).

The constant $C_{1}$ characterizes a rigid translation.

Let us consider now the problem $P^{(3)}$ defined by $(7.7)$ and $(7.8)$. We seek the solution of (7.7) as functions only of $r$. We obtain [12]

$$
\begin{aligned}
& u_{r}^{(3)}=E_{1} r, u_{\theta}^{(3)}=\frac{e^{(2)}}{\delta^{(2)}} E_{2} I_{1}\left(\delta^{(2)} r\right), \varphi_{z}^{(3)}=E_{2} I_{o}\left(\delta^{(2)} r\right), 0 \leq r \leq r_{2}, \\
& u_{r}^{(3)}=F_{1} r+F_{3} r^{-1}, u_{\theta}^{(3)}=\frac{e^{(1)}}{\delta^{(1)}}\left[F_{2} I^{1}\left(\delta^{(1)} r\right)-F_{4} K_{1}\left(\delta^{(1)} r\right)\right]+F_{5} r^{-1}, \\
& \varphi_{2}^{(3)}=F_{2} I_{o}\left(\delta^{(1)} r\right)+F_{4} K_{o}\left(\delta^{(1)} r\right), \quad r_{2} \leq r \leq r_{1},
\end{aligned}
$$

where $E_{1}, E_{2}, F_{1}, \ldots, F_{4}$ are unknown constants. If we introduce the displacements $(7.17)$ in (7.8), we obtain the solution of the problem $P^{(3)}$ in the form

$$
\begin{aligned}
& u_{r}^{(3)}=E_{1} r, u_{\theta}^{(3)}=0, \varphi_{z}^{(3)}=0, \quad 0 \leq r \leq r_{2}, \\
& u_{r}^{(3)}=F_{1} r+F_{3} r^{-1}, u_{\theta}^{(3)}=0, \varphi_{z}^{(3)}=0, \quad r_{2} \leq r \leq r_{1},
\end{aligned}
$$

where

$$
\begin{aligned}
& F_{3}=\left(\nu^{(1)}-\nu^{(2)}\right)\left\{\frac{2 \mu^{(1)}+\kappa^{(1)}}{2 \lambda^{(2)}+2 \mu^{(2)}+\kappa^{(2)}}\left(r_{2}^{-2}-r_{1}^{-2}\right)+r_{1}^{-2}+r_{2}^{-2}-2 \nu^{(1)} r_{1}^{-2}\right\}^{-1}, \\
& F_{1}=-\nu^{(1)}+\left(1-2 \nu^{(1)}\right) F_{3} r_{1}^{-2}, E_{1}=-\nu^{(1)}+\left[r_{2}^{-2}+r_{1}^{-2}-2 \nu^{(1)} r_{1}^{-2}\right] F_{3}, \\
& E_{2}=F_{2}=F_{4}=0, \nu^{(\rho)}=\lambda^{(\rho)} /\left(2 \lambda^{(\rho)}+2 \mu^{(\rho)}+\kappa^{(\rho)}\right) .
\end{aligned}
$$

The solution for the extension and bending of the composite circular cylinder is given by

$$
\begin{aligned}
& u_{r}=\left(a_{1} \cos \theta+a_{2} \sin \theta\right)\left(-\frac{1}{2} z^{2}-\frac{1}{8} r^{2}+v_{r}^{o}\right)+a_{3} u_{r}^{(3)}, \\
& u_{\theta}=\left(a_{1} \sin \theta-a_{2} \cos \theta\right)\left(\frac{1}{2} z^{2}-\frac{5}{8} r^{2}+v_{\theta}^{o}\right), \\
& u_{z}=\left(a_{1} \cos \theta+a_{2} \sin \theta\right) r z+a_{3} z, \\
& \varphi_{r}=-\left(a_{1} \sin \theta-a_{2} \cos \theta\right) z, \\
& \varphi_{\theta}=-\left(a_{1} \cos \theta+a_{2} \sin \theta\right) z \\
& \varphi_{z}=\left(a_{1} \sin \theta-a_{2} \cos \theta\right)\left(-r+\psi_{z}^{o}\right),
\end{aligned}
$$

where $v_{r}^{o}, v_{\theta}^{o}, \psi_{z}^{o}$ are given by $(7.14), u_{r}^{(3)}$ is given by $(7.18)$ and the constants $a_{i}(i=1,2,3)$ can be determined from the system (3.7). From (7.16), (7.18) and (3.8) it follows

$$
\begin{aligned}
A_{12}= & A_{21}=0, A_{3 \alpha}=A_{\alpha 3}=0, \\
A_{33}= & \pi\left(\lambda^{(2)}+2 \mu^{(2)}+\kappa^{(2)}+2 \lambda^{(2)} E_{1}\right) r_{2}^{2}+\pi\left(\lambda^{(1)}+2 \mu^{(1)}+\kappa^{(1)}+2 \lambda^{(1)} F_{1}\right)\left(r_{1}^{2}-r_{2}^{2}\right), \\
A_{11}= & A_{22}=\pi\left(2 \mu^{(2)}+\kappa^{(2)}\right) r_{2}^{4} / 4+\pi\left(\beta^{(2)}+\gamma^{(2)}\right) r_{2}^{2}-\pi \beta^{(2)} r_{2}\left[C_{3} I_{1}\left(\delta^{(2)} r_{2}\right)\right. \\
& \left.-8 C_{2} r_{2}\right]-2 \pi \lambda^{(2)}\left(2 c^{(2)}-d^{(2)}\right) C_{2} r_{2}^{4}+\pi \lambda^{(1)} D_{5}\left(r_{1}-r_{2}\right) \\
& +\pi\left(2 \mu^{(1)}+\kappa^{(1)}\right)\left(r_{1}^{4}-r_{2}^{4}\right) / 4+\pi\left(\beta^{(1)}+\gamma^{(1)}\right)\left(r_{1}^{2}-r_{2}^{2}\right)-\pi \beta^{(1)}\left\{D _ { 3 } \left[r_{1} I_{1}\left(\delta^{(1)} r_{1}\right)\right.\right. \\
& \left.-r_{2} I_{1}\left(\delta^{(1)} r_{2}\right)\right]-8 D_{2}\left(r_{1}^{2}-r_{2}^{2}\right)+D_{4}\left[r_{1} K_{1}\left(\delta^{(1)} r_{1}\right)-r_{2} K_{3}\left(\delta^{(1)} r_{2}\right)\right]-2 \pi \lambda^{(1)}\left(2 c^{(1)}-d^{(1)}\right) D_{2}\left(r_{1}^{4}-r_{2}^{4}\right),
\end{aligned}
$$


so that we obtain

$$
a_{1}=\frac{1}{A_{11}} M_{2}, a_{2}=-\frac{1}{A_{11}} M_{1}, a_{3}=-\frac{1}{A_{33}} P_{3} .
$$

From the above results it is easy to derive the solution in the case of homogeneous cylinders.

[1] D. IEŞAN, Int. J. Engng Sci. 9. 1047 (1971).

[2] D. IESAN, Almansi's problem in micropolar elasticity. Int. J. Engng Sci. 12, 361 (1974).

[3] D. IEŞAN, ZAMM, 58, 95 (1978).

[4] D. IEŞAN, Int. J. Engng Sci. 9,879 (1971).

[5] C. USIDUS and M. SOKOLOWSKI: Bull. Acad. Polon. Sci. Sér. Sci. Techn. 21, 19 (1973).

[6] G. V. KRISHNA REDDY and N. K. VENKATASUBRAMANIAN, Int. J. Engng Sci. 14, 1047 (1976).

[7] D. IESAN, Rozpr. Inzyn. 24, 289 (1976).

[8] D. IEŞAN, Ann. Mat. Pura Appl. (IV), 108, 149 (1976).

[9] A. C. ERINGEN, Fracture, Vol. 2. Academic Press, New York (1967).

[10] G. FICHERA, In Handbuch der Physik, Vol. VIa/2. Springer, New York (1972).

[11] C. M. IESAN, Atti. Accad. Sci. Torino, Cl. Sci. Fis. Mat. Natur. 108, 657 (1974).

[12] B. M. CHIU and I. D. LEE, Int. J. Engng Sci. 11, 997 (1973).

(13) A. C. SMITH, Recent Advances in Engng Sci. (Edited by A. C. Eringen), Vol. 5/II, p. 129. Gordon \& Breach, London (1970) 